\title{
Densidad básica de la madera de pino oregón y su relación con las condiciones de crecimiento en la Patagonia Andina Argentina
}

\author{
Wood basic density of the Douglas fir and its relation with growth \\ conditions in the Patagonian Andes, Argentina
}

\author{
MIGUEL M. DAVEL ${ }^{1-2}$, ALEJANDRO JOVANOVSKI ${ }^{1-2}$, DIEGO MOHR BELL 2 \\ ${ }^{1}$ Centro de Investigación y Extensión Forestal Andino Patagónico (CIEFAP) CC 14, CP9200, \\ Esquel, Chubut, Argentina. Tel/Fax: +54 2945 453948. E-mail: mdavel@ciefap.cyt.edu.ar \\ ${ }^{2}$ Depto. Ingeniería Forestal, Universidad Nacional de la Patagonia San Juan Bosco, \\ Sede Esquel, Ruta 259 Km4, CP9200, Esquel, Chubut, Argentina.
}

\begin{abstract}
SUMMARY
Wood density of Douglas fir (Pseudotsuga menziesii (Mirb) Franco) growing in Patagonia Argentina and its relation with site conditions and dominant tree growth were studied. Wood samples were obtained from 84 dominant trees coming from 42 plantations located across the Andean region of the provinces of Neuquén, Río Negro and Chubut which constitute the distribution area of Douglas fir in Argentina. The mean mature wood density of Douglas Fir was $0,381 \mathrm{~g} / \mathrm{cm}^{3}$. In each studied plantation, information about topographic, edaphic, climatic and stand variables were obtained. The relation among these variables and wood density was evaluated with multiple regression analyses. Results show that rings-width and site index were significantly related with specific gravity, and explained 52\% of this attribute's variation. Results also showed that an inverse relationship exist between site index and wood density: Better site index produce timber that has less wood density.
\end{abstract}

Key words: Patagonia, Douglas-fir, wood density, site index, growth ring width.

\section{RESUMEN}

Se estudió la densidad básica de la madera de pino oregón (Pseudotsuga menziesii (Mirb) Franco) creciendo en la Patagonia Andina Argentina y su relación con las condiciones de sitio y el crecimiento de los árboles dominantes del rodal. Las muestras se obtuvieron de 84 árboles dominantes provenientes de 42 plantaciones de la zona andina de las provincias de Neuquén, Río Negro y Chubut, la cual constituye el área de mayor aptitud para forestar con esta especie en Argentina. La densidad básica media de la madera madura para la población analizada fue de $0,381 \mathrm{~g} / \mathrm{cm}^{3}$. En cada una de las plantaciones muestreadas se obtuvo información sobre variables topográficas, edáficas, climáticas y de rodal. La relación entre la densidad básica y estas variables se evaluó mediante un análisis de regresión múltiple. De las variables analizadas, el índice de sitio y el ancho de los anillos de crecimiento se relacionaron significativamente con la densidad básica, explicando en conjunto el $52 \%$ de la variación de la misma en la población analizada. La relación encontrada indica que a mejor productividad del sitio y mayor tasa de crecimiento, menor será la densidad de la madera obtenida, tendencia que debiera ser considerada en los esquemas de manejo que se propongan para la especie.

Palabras clave: Patagonia, pino oregón, densidad de la madera, índice de sitio, ancho anillos de crecimiento. 


\section{INTRODUCCION}

El pino oregón (Pseudotsuga menziesii (Mirb.) Franco) presenta excelentes crecimientos en la Patagonia Andina Argentina, alcanzando para sitios de productividad media a alta de 20 a $36 \mathrm{~m}^{3} /$ ha año (1). Esta especie es muy apreciada en la región no sólo por sus crecimientos, sino también por las características de su madera, su sanidad y la calidad de los productos que pueden obtenerse a partir de ella. Posee una madera resistente y estable que tiene amplias aplicaciones en estructuras, pisos, muebles, aberturas, chapas, terciados, terminaciones de interiores y pulpa para papel, entre otras (2).

Dentro de las propiedades físicas de la madera, la densidad es una de las más importantes, ya que determina el valor y la utilidad de la misma, y está fuertemente correlacionada con otras propiedades como la resistencia mecánica, la rigidez, la conductividad térmica y el calor específico (3).

Diversos factores, tales como características hereditarias, ambientales y estructura del bosque afectan los patrones de crecimiento de los árboles y a su vez determinan la morfología y la composición química de las células y, por lo tanto, condicionan la densidad de la madera. En este sentido, las opiniones acerca del modo en que la calidad de sitio y los tratamientos silvícolas afectan la calidad de la madera de pino oregón son diversas y en ciertos casos muy contradictorias.

Zobel y van Buijtenen (4) mencionan que la variación de la densidad de la madera entre árboles de pino oregón debido a causas genéticas es tan grande, que no permite apreciar la variabilidad por causa de otros factores externos como calidad de sitio, clase de copa y ubicación geográfica.

Sin embargo, Cown (5) reporta que existe una relación inversa entre la calidad de sitio y la densidad de la madera. Resultados similares fueron obtenidos por Carlson y Nimlos (6), quienes informan diferencias en la densidad para distintos tipos de suelo.

Otro efecto relevante, y también controvertido, es el que tienen las prácticas silviculturales sobre las propiedades de la madera de pino oregón. Por ejemplo, existen distintas opiniones acerca del efecto de los raleos sobre la densidad. Parker et al. (7) sostienen que la densidad de la madera no fue afectada significativamente por los raleos, a pesar de que producen un aumento de la tasa de crecimiento en diámetro. Por otro lado, Erickson y Harrison (8) encontraron que los raleos producen madera de menor densidad, fundamentalmente en los 3 a 4 años después de aplicado el tratamiento. Hapla (9) y Lausberg et al. (10) encontraron que elevadas tasas de crecimiento provocadas por mayores espaciamientos producen una disminución significativa en la densidad de la madera.

Las podas parecen tener el efecto opuesto, produciéndose madera de mayor densidad por debajo de la copa remanente luego de efectuadas las mismas. En general, la poda adelanta el cese de la producción de madera juvenil e incrementa la formación de leño tardío, aumentando consecuentemente la densidad. Megraw (11) encontró que la poda produce un incremento en la densidad de la madera por debajo de la copa remanente para la especie en estudio. Gartner et al. (12), en cambio, no encontraron efectos de la posición de la copa sobre la transición de madera juvenil a madera madura y, por lo tanto, tampoco sobre la densidad.

El conocimiento de la variabilidad en las propiedades físicas y mecánicas es de fundamental importancia para los usuarios de la madera al momento de evaluar sus múltiples aplicaciones y, para los silvicultores, al momento de fijar los objetivos de producción en términos de manejo. Esto permite optimizar ciertas propiedades de acuerdo a las necesidades industriales y disminuir, dentro de ciertos límites, la heterogeneidad de la materia prima (13).

En la región patagónica argentina la superficie forestada con pino oregón es aún pequeña, y la mayoría de las plantaciones existentes carecen de intervenciones silviculturales. Por otro lado, las condiciones ambientales de los sitios donde se planta la especie son variables y están regidas principalmente por un gradiente de precipitación muy fuerte. Desde los distintos organismos técnicos se proponen esquemas de manejo que persiguen la obtención de madera libre de nudos maximizando el crecimiento, sin embargo, se desconoce la calidad del producto que se obtendrá. En este contexto se realizó el presente trabajo que tuvo como objetivo principal estudiar la relación de la densidad básica de la madera de pino oregón en diferentes condiciones de sitio y crecimiento en la Patagonia Argentina. 


\section{MATERIAL Y METODOS}

Area de estudio. Esta abarca el área donde se foresta con pino oregón en la región andina de las provincias de Neuquén, Río Negro y Chubut en la República Argentina. Dicha área se extiende aproximadamente desde los $40^{\circ}$ a los $43^{\circ}$ de latitud sur y entre los $71^{\circ}$ y $71^{\circ} 40^{\prime}$ de longitud oeste (figura 1) y se caracteriza por presentar un gradiente edáfico y de precipitación oeste-este muy importante (1). Dentro de esta región, hay definidas dos zonas de crecimiento para la especie en base a rangos de precipitación y a diferencias en los patrones de crecimiento y productividad. El límite entre ambas zonas está definido por la isohieta de los $1.000 \mathrm{~mm}$ (14).

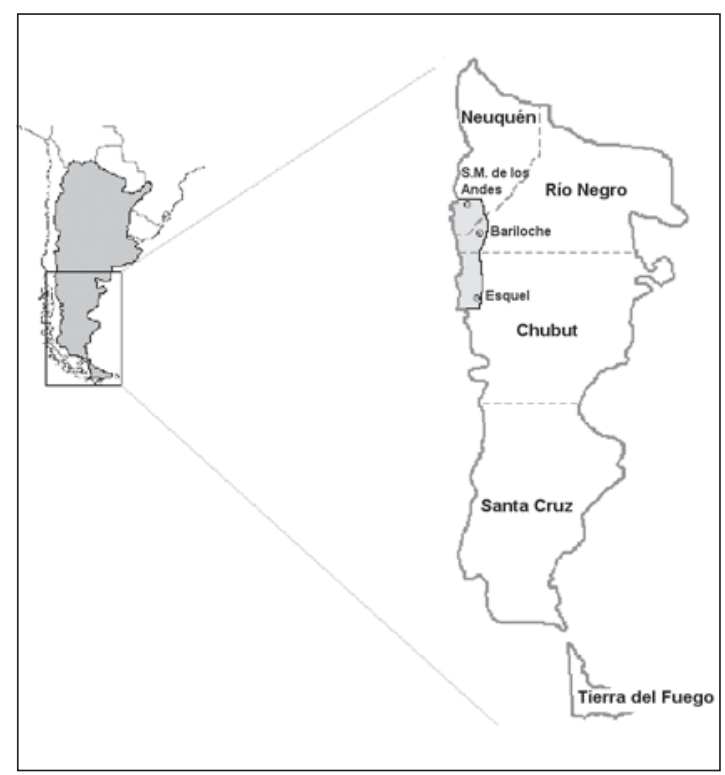

Figura 1. Area de aptitud forestal para plantaciones de pino oregón en la Patagonia Andina Argentina.

Forest aptitude area for Douglas fir plantations in the Argentinean Patagonian Andes.

Toma de las muestras. Las plantaciones muestreadas para el presente estudio se seleccionaron tratando de cubrir las variaciones de sitio, edad (mayores a 10 años) y manejo de la población analizada. En cada una de las plantaciones seleccionadas se instaló una parcela circular de $200 \mathrm{~m}^{2}$, en las que entraron entre 15 y 45 árboles por parcela. En cada parcela se voltearon los dos árboles dominantes más gruesos. En total se cortaron 84 árboles provenientes de 42 plantaciones de la región. Para el presente estudio se utilizaron sólo los que presentaban madera madura, quedando 56 árboles provenientes de 28 rodales. De cada uno de estos árboles se cortó un disco transversal de madera libre de nudos u otras anomalías a la altura de $1,30 \mathrm{~m}$ desde el suelo (diámetro a la altura del pecho o DAP).

A partir de cada disco se cortó un listón de un largo equivalente al diámetro de dicho disco, de $2,5 \mathrm{~cm}$ de ancho por $1 \mathrm{~cm}$ de espesor, incluyendo la médula. Cada listón fue cortado en forma de cuña, en ambos radios, y luego dividido en probetas conteniendo 3 anillos de crecimiento desde la corteza a la médula (figura 2). Las probetas se cortaron de esta forma para disminuir la influencia de variaciones climáticas anuales. En caso que los anillos de crecimiento fueran muy delgados se obtuvieron probetas que contenían más de 3 anillos. Para el estudio se consideró sólo la madera madura, por lo que las probetas ubicadas entre la médula y los 14 años fueron descartadas. Se consideró esta edad en base a estudios realizados por Jovanovski et al. (15). En total se midieron 136 probetas.

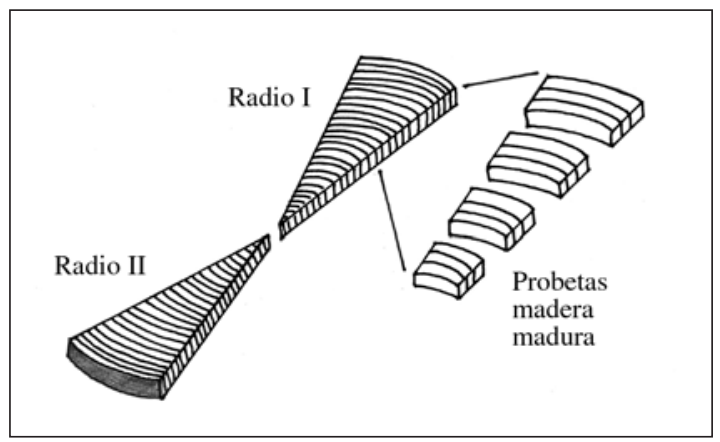

Figura 2. Listones en forma de cuña y obtención de probetas de densidad.

Cutting method to determine wood density samples.

Determinación de la densidad básica. Debido a que el material de ensayo se encontraba a un contenido de humedad del $8-10 \%$, para la determinación de la densidad básica, se decidió que, en primera instancia, las probetas fueran colocadas en estufa a $103 \pm 2{ }^{\circ} \mathrm{C}$ hasta peso constante, de tal manera de obtener la masa anhidra. Posteriormente, se determinó el volumen saturado por desplazamiento de agua, para lo cual las probetas fueron 
previamente sumergidas en agua con aplicación de vacío (100 milibares) hasta un contenido de humedad superior a $150 \%$, procedimiento que no afecta la máxima hinchazón requerida para la estimación del volumen (16). Tanto para la estimación de la masa como del volumen se utilizó una balanza electrónica de precisión 0,001 g. A partir de estos valores se obtuvo la densidad básica con la ecuación [1].

$$
D B=M a / V v
$$

donde:

DB: densidad básica

Ma: masa de la madera en estado anhidro Vv: volumen de la madera en estado verde

Para este trabajo se utilizó como valor de densidad básica el promedio obtenido de las probetas (de ambos radios) de madera madura de los árboles de cada parcela.

Variables ambientales y de rodal. Las características ambientales y de rodal se midieron en las mismas parcelas donde se voltearon los árboles analizados. Las variables consideradas fueron las siguientes:

- Topográficas: latitud y longitud (en grados, minutos y segundos, determinadas con GPS Garmin 75 con una precisión en XY de 100 m), altitud (en metros sobre el nivel del mar, determinada con altímetro marca Thommen, precisión $\pm 10 \mathrm{~m}$ ), pendiente (medida con clinómetro Suunto en grados) y exposición (medida con brújula Suunto en grados), posición en la pendiente (valle, ladera baja, media ladera, ladera alta, cumbre).

- Climáticas: se consideró la precipitación media anual en milímetros tomada a partir de mapas de isohietas $(17,18)$ o de datos de estaciones meteorológicas cercanas en caso de haberlas.

- Edáficas: profundidad total (en metros, medida con barreno de suelos desde la superficie hasta algún impedimento físico como capa de arcilla, roca, etc., o hasta 1 metro), textura (estimada a campo). Además se extrajeron muestras de la parte inferior del horizonte A con el fin de determinar contenido de materia orgánica en laboratorio. Los análisis fueron realizados por el Laboratorio de Suelos de la
Facultad de Cinco Saltos de la Universidad Nacional del Comahue. Se consideró el contenido de materia orgánica por haber sido la variable ambiental más relacionada con la productividad de la especie según estudios realizados en la región (19).

- De rodal: edad en años, índice de sitio (estimado a partir de las funciones de sitio ajustadas por Davel y Ortega (14) para una edad de referencia de 20 años), el IDR (índice de densidad de rodal de Reineke) y ancho de anillos (AA) promedio por parcela. Esta última variable se consideró como indicadora del efecto de la densidad de rodal sobre el crecimiento en diámetro.

Los datos de latitud y longitud fueron transformados para el análisis en valores centesimales. La pendiente y la exposición se consideraron combinadas a partir del método de Stage (20), que permite estimar matemáticamente cuáles son las exposiciones, combinadas con las pendientes, más favorables para la expresión del crecimiento que se desea predecir. Los datos cualitativos (posición sobre la pendiente y textura) debieron ser codificados para realizar el análisis. En el caso de la posición en la pendiente se utilizó el criterio propuesto por Carvell y Tryon (21), que le asigna números del 1 al 5 de acuerdo a si la posición es en el valle $\{1\}$, ladera baja $\{2\}$, media ladera $\{3\}$, ladera alta $\{4\}$, o si es sobre cumbre $\{5\}$. En el caso de la textura se codificó de la siguiente manera: textura gruesa $\{3\}$, texturas medias $\{6\}$ y texturas finas $\{9\}$. En el cuadro 1 se presentan los estadísticos descriptivos de la base de datos utilizada para el análisis.

Análisis de la información. En un primer análisis se graficaron las variables independientes de a pares para determinar qué tipo de relaciones existían entre ellas y cuáles presentaban correlación significativa entre sí. Esto se efectuó con el propósito de evitar incluir en los modelos variables independientes significativamente correlacionadas. Luego se evaluó, mediante análisis de regresión simple, la relación individual de cada variable con la densidad básica para conocer el grado de relación existente y estudiar los residuales de cada variable por separado.

Las variables seleccionadas se utilizaron como variables independientes en una regresión múltiple aplicando la técnica "stepwise", para construir 


\section{CUADRO 1}

Media, desvío estándar, máximo y mínimo de densidad básica (DB), variables ambientales y de rodal analizadas. (IS = índice de sitio; IDR: índice de densidad de rodal de Reineke; $\mathrm{MO}=$ materia orgánica; $\mathrm{AA}=$ ancho de anillos medio por parcela).

Mean, standard deviation, maximum and minimum values of wood basic density (DB) and environmental and stand variables analysed (IS = site index; IDR= Reineke's stand-density index; $\mathrm{MO}=$ organic matter; $\mathrm{AA}=$ mean ring width per plot)

\begin{tabular}{|c|c|c|c|c|}
\hline Variable & Media & Desv. Est. & Máximo & Mínimo \\
\hline $\mathrm{DB}\left(\mathrm{g} / \mathrm{cm}^{3}\right)$ & 0,381 & 0,040 & 0,435 & 0,310 \\
\hline Edad (años) & 25 & 7,6 & 52 & 17 \\
\hline IS & 15,2 & 2,3 & 20,9 & 10,5 \\
\hline IDR & 980 & 346 & 1.894 & 431 \\
\hline Pendiente $(\%)$ & 13,6 & 8,1 & 34 & 0 \\
\hline Exposición $\left(^{\circ}\right)$ & 143,4 & 91,9 & 330 & 0 \\
\hline Profundidad de suelo (m) & 0,77 & 0,15 & $>1$ & 0,5 \\
\hline Textura* & - & - & 9 & 3 \\
\hline $\mathrm{MO}(\%)$ & 7 & 4,4 & 17,2 & 2,1 \\
\hline Precipitación media anual (mm) & 1.075 & 541 & 2.361 & 600 \\
\hline Latitud $\left({ }^{\circ}\right)$ & - & - & $42^{\circ} 2^{\prime} 7^{\prime}$, & $40^{\circ} 74^{\prime}$, \\
\hline Longitud $\left({ }^{\circ}\right)$ & - & - & $71^{\circ} 36^{\prime} 3^{\prime \prime}$ & $71^{\circ} 2^{\prime} 45^{\prime \prime}$ \\
\hline Altitud (m) & 823 & 225 & 1.124 & 370 \\
\hline $\mathrm{AA}(\mathrm{mm})$ & 5,8 & 1,2 & 8,1 & 3,1 \\
\hline
\end{tabular}

* En el caso de la textura la moda es 6

una ecuación de estimación de la densidad básica. Por medio de este método se fueron seleccionando aquellas variables que presentaron una correlación significativa con la densidad básica, a un nivel de probabilidad del $5 \%$, que fueran objetivas desde el punto de vista práctico y consistentes desde el punto de vista biológico. Entre dos variables explicatorias con alta correlación entre sí y significativamente relacionadas con la densidad básica, se eligió para el modelo aquella que explicara mejor las variaciones de la densidad básica. El modelo general utilizado fue el siguiente [2]:

$$
\hat{Y}=b_{0}+b_{1} X_{1}+b_{2} X_{2}+\cdots \cdots \cdots+b_{k} X_{k}
$$

donde:

$Y$ : valor esperado de la variable dependiente $\mathrm{b}_{0}$ : intercepto de $\mathrm{Y}$

$\mathrm{X}_{\mathrm{j}}$ : variable independiente $(\mathrm{j}=1,2, \ldots \ldots, \mathrm{k})$

$\mathrm{b}_{\mathrm{i}}$ : parámetros a ser estimados $(\mathrm{i}=1,2, \ldots \ldots, \mathrm{k})$
La calidad del ajuste fue analizada a través del coeficiente de determinación $\left(\mathrm{R}^{2}\right)$ que indica el porcentaje de la variación total explicado por la regresión. También se analizaron los gráficos de residuales para detectar valores extremos que pudieran indicar errores en la base de datos o tendencias anormales que acusaran heterogeneidad de la varianza, considerándose una distribución satisfactoria aquella que se asemeja a una banda horizontal (22).

La evaluación de la bondad de predicción del modelo se realizó utilizando la misma base de datos usada para el ajuste, ya que no se contó con información adicional. Se compararon los valores observados con los estimados por el modelo, utilizándose como medida de error la raíz del error cuadrático medio (RECM) [3] y como medida de sesgo, la diferencia agregada (DIFA) [4]:

$$
R E C M=\sqrt{\sum(o-e)^{2} / n}
$$




$$
D I F A=\sum(o-e) / n
$$

donde:

$\mathrm{o}=$ observado

$\mathrm{e}=$ estimado

$\mathrm{n}=$ número de observaciones

Mediante la división por la media de los valores observados (Ô) y su multiplicación por $100 \mathrm{se}$ obtuvieron los valores porcentuales [5] y [6]:

$$
\begin{aligned}
R E C M \% & =(R E C M * 100) / \hat{O} \\
D I F A \% & =(|D I F A| * 100) / \hat{O}
\end{aligned}
$$

\section{RESULTADOS Y DISCUSION}

Relación individual de cada variable con la densidad básica $(D B)$. Al analizar las variables por separado las dos que mejor relación presentaron con la DB fueron el AA $\left(\mathrm{R}^{2}=0,46, \mathrm{p}<0,0001\right)$ y el IS $\left(R^{2}=0,30, p=0,001\right)$. Ninguna de las otras variables analizadas presentó una relación significativa con la densidad básica de la madera.

El AA presentó una relación lineal inversa con la densidad básica (figura 3), es decir, que a mayores densidades de rodal y menor AA, la DB de la madera fue mayor y viceversa. El rango de la densidad de los rodales analizados, expresada como densidad relativa a través del IDR, se puede observar en el cuadro 1. Esta relación es semejante a la citada por Hapla (9) y Lausberg et al. (10) para la especie en estudio.

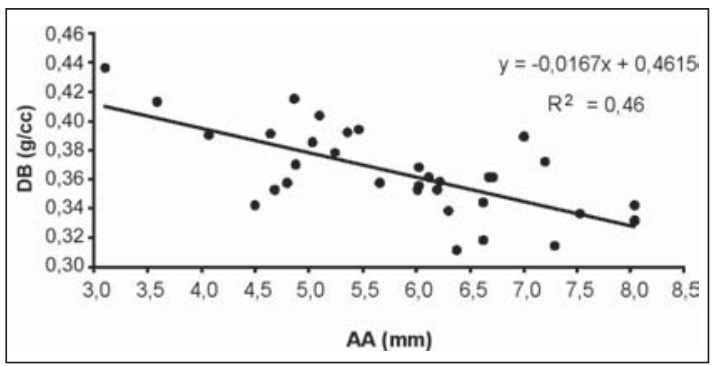

Figura 3. Relación de la densidad básica (DB) con el ancho de los anillos de crecimiento (AA). Se presentan los parámetros de la ecuación y el coeficiente de determinación $\left(\mathrm{R}^{2}\right)$ de la misma.

Wood basic density (DB) and growth ring width (AA) relation. The parameters of the equation and the coefficient of determination of this model are presented.
El IS también presentó una relación lineal inversa con la $\mathrm{DB}$, lo que indica que a mejores sitios la DB de la madera producida fue menor (figura 4), lo que concuerda con lo citado por Cown (5) para el pino oregón. Varios estudios muestran una relación inversa entre la proporción del leño tardío (PLT) y la precipitación y la disponibilidad de agua para pino oregón (4). Por otro lado, la relación entre la PLT y la densidad de la madera es directamente proporcional. Kollman y Coté (23) indican que a medida que aumenta la PLT la densidad crece, esto mismo fue observado por Jovanovski et al. (15) para pino oregón en Patagonia. Esto podría explicar la relación observada entre la DB de la madera y el IS para la especie en esta región.

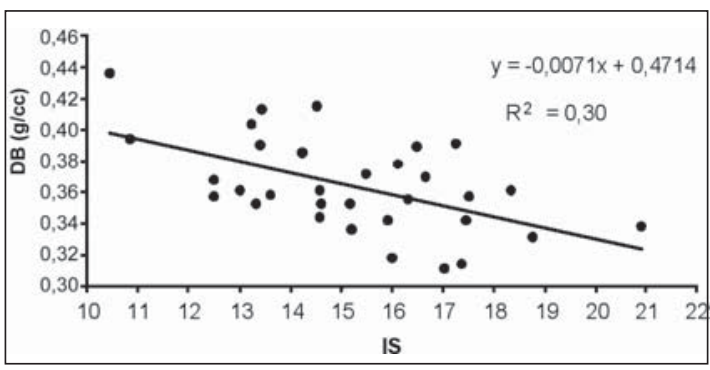

Figura 4. Relación de la densidad básica (DB) con el índice de sitio (IS). Se presentan los parámetros de la ecuación y el coeficiente de determinación $\left(\mathrm{R}^{2}\right)$ de la misma.

Wood basic density (DB) and site index (IS) relation. The parameters of the equation and the coefficient of determination of this model are presented.

Observando la relación de la DB con el IS, se puede deducir que esta última variable es un factor determinante de la densidad. Por otro lado, de la relación con el AA se infiere que la regulación del crecimiento en diámetro por medio de prácticas silviculturales constituye una herramienta que permite influir sobre la densidad de la madera a producir.

A modo ilustrativo, en las figuras 5 y 6 se muestra el efecto que tienen los raleos sobre el AA y la DB, donde ésta cae en los años próximos siguientes de efectuarse la intervención como consecuencia de un aumento en el crecimiento diametral acusado por el incremento en el AA. Este resultado coincide con el obtenido por Erickson y Harrison (8), quienes encontraron que los raleos produjeron madera de menor densidad en los 3 a 4 años siguientes a la aplicación del tratamiento. 
Construcción del modelo de regresión múltiple. De todas las variables analizadas, sólo el IS y el AA presentaron una relación significativa con la DB al 5\% de probabilidad. El ajuste del modelo fue altamente significativo y ambas variables en conjunto explicaron el $52 \%$ de la variación de la DB. La expresión matemática del modelo ajustado es la siguiente [7]:

$$
\boldsymbol{D B}=0,49594-0,01312 \boldsymbol{A A}-0,00368 \boldsymbol{I S} \quad\left(\mathrm{R}^{2}=0,52, \mathrm{p}<0,0001\right)
$$

La distribución de los residuales resultó satisfactoria, no evidenciándose tendencias anómalas ni heterogeneidad de la varianza, por lo que no fue necesario realizar ninguna transformación de las variables (figura 7).

Las estimaciones de la DB de la madera a partir del IS y el AA presentaron valores muy bajos de error $(\mathrm{RECM}=5,6 \%)$ y sesgo (DIFA= $0,023 \%$ ); sin embargo, el modelo realiza una leve sobreestimación de la DB, reflejada en el valor negativo de la DIFA.

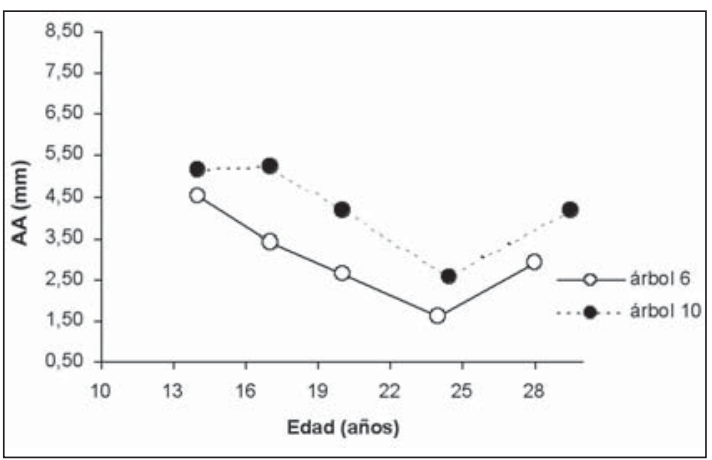

Figura 5. Efecto del raleo realizado a los 24 años de edad sobre el AA de dos árboles del mismo rodal.

Effects of thinning, at 24 years of age, over the growth ring width in two trees of the same stand.

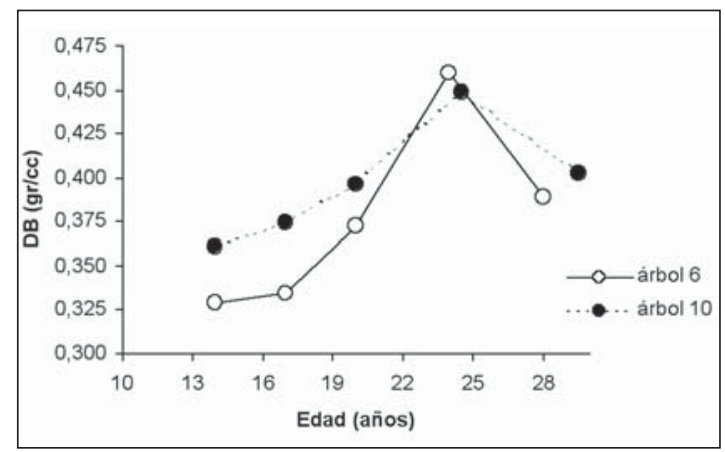

Figura 6. Efecto del raleo realizado a los 24 años de edad sobre la DB de dos árboles del mismo rodal.

Effects of thinning, at 24 years of age, over the basic density in two trees of the same stand.

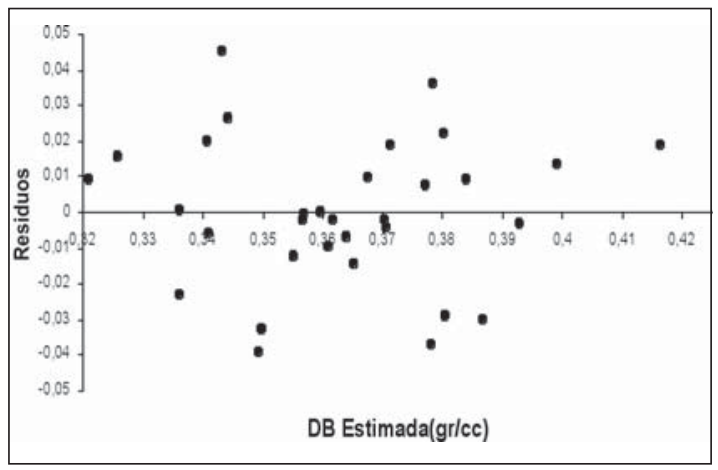

Figura 7. Gráfico de residuales del modelo seleccionado para predecir la DB a partir del índice de sitio y el ancho de los anillos de crecimiento.

Residual plots of the model selected to predict wood basic density with the site index and the growth ring width.

\section{CONCLUSIONES}

Dentro de las variables analizadas, el índice de sitio (IS) y el ancho de anillos (AA) fueron las que presentaron una mayor relación con la densidad básica de la madera del pino oregón creciendo en Patagonia. El AA por sí sólo explica el $46 \%$ de la variación encontrada en la densidad básica entre los distintos rodales estudiados, mientras que el IS explica un 30\% de esta variación. Ambas variables tienen una relación inversa con la densidad básica, y explicaron en conjunto el $52 \%$ de la variación de la DB en la región.

Esta relación indica que a mejor productividad y mayor tasa de crecimiento en diámetro, menor será la DB de la madera obtenida. La función desarrollada constituye una herramienta que puede ser empleada en la optimización de los esquemas de manejo en función de los objetivos de producción perseguidos. Esta función permitiría, conociendo el IS del rodal, establecer cómo se debe manejar la densidad del mismo para obtener un determinado AA y así lograr madera con una DB cercana a la deseada. 


\section{BIBLIOGRAFIA}

(1) DAVEL, M. Identificación y caracterización de zonas de crecimiento para pino oregón en la Patagonia Andina Argentina. Tesis para optar por el grado de Magíster en Ciencias Mención Manejo Sustentable de Recursos Forestales. Universidad Austral de Chile. Valdivia, Chile, 1998, $120 \mathrm{p}$.

(2) INFOR. Monografía de pino oregón. Pseudotsuga menziesii. Potencialidad de especies y sitios para una diversificación silvícola nacional. Proyecto financiado por CONAF. Santiago, Chile, 1996, 128 p.

(3) TUSET, R., F. DURAN. Manual de maderas comerciales, equipos y procesos de utilización. Aserrado, secado, preservación, descortezado, partículas. Editorial Hemisferio Sur. Montevideo, Uruguay, 1974, 688 p.

(4) ZOBEL, B.J., J.P. van BUIJTENEN. Wood variation. Its causes and control. Springer-Verlag. Springer Series in Wood Sciences. Berlin, Germany, 1989, 363 p.

(5) COWN, D.J. New Zealand radiata pine and Douglasfir: Suitability for processing. New Zealand Ministry of Forestry, Forest Research Institute, FRI Bulletin, 1992, $\mathrm{N}^{\circ} 168$.

(6) CARLSON, T.C., T.J. NIMLOS. Using soil series to predict site index and wood specific gravity in Western Montana. Northwest Sci, 1966, vol. 40, No 2, p. 56-57.

(7) PARKER, M.L., K. HUNT, W. WARREN, R. KENNEDY. Effect of thinning and fertilization on intraring characteristics and kraft pulp yield of Douglas-fir. Appl. Polym Symp, 1976, No 28, p. 1095-1086.

(8) ERICKSON, H.D., A.T. HARRISON. Douglas-fir wood quality studies. Effects of age and simulated growth on wood density and anatomy (Part I). Wood Sci Technol, 1974, No 8 , p. 207-226.

(9) HAPLA, F. How to bring into accord the silvicultural management and end-users' interests in case of the Douglas fir. Timber management toward wood quality and end-product value. CTIA / IUFRO International Wood Quality Workshop, 1997. Quebec. Canada. p. 3-7.

(10) LAUSBERG, M.J., D.J. COWN, D.L. McCONCHIE, J.H. SKIPWITH. Variation in some wood properties of Pseudotsuga menziesii provenances grown in New Zealand. New Zealand of Forestry Science, 1995, vol. 25, No 2, p. 133-146.

(11) MEGRAW, R.A. Effect of silvicultural practices on wood quality. In Proceedings: TAPPI R\&D Conference,
Raleigh, North Carolina Technical Association of the Pulp and Paper Industry, Atlanta, 1986. p. 27-34.

(12) GARTNER, B.L., E.M. NORTH, G.R. JOHNSON, R. SINGLETON. Effects of live crown on vertical patterns of wood density and growth in Douglas-fir. Can. J. For. Res., 2002, vol. 32, p. 439-447.

(13) DELMASTRO, R., J. DIAZ-VAZ, J.SCHLATTER. Variabilidad de las características tecnológicas hereditarias del Pinus radiata (D. Don). Informe $\mathrm{N}^{\circ} 3$. Chile. Universidad Austral de Chile, 1981, 187 p.

(14) DAVEL, M.M., A. ORTEGA. Productividad por zonas de crecimiento para pino oregón (Pseudotsuga menziesii (Mirb.) Franco) en la Patagonia Andina Argentina. Inves. Agr.: Sist. Recur. For. (España), 2003, vol. 12, № 3, p. 33-45.

(15) JOVANOVSKI, A., M.M. DAVEL, D. MOHR BELL. Densidad básica de la madera de Pseudotsuga menziesii (Mirb.) Franco en la Patagonia. Inves. Agr.: Sist. Recur. For. (España), 2005, vol. 14, No 2, p. 153-160.

(16) KOLLMANN, F. Tecnología de la madera y sus aplicaciones. Instituto Forestal de Investigaciones, Experiencias y Servicios de la Madera. Tomo Primero. España, 1959. 675p.

(17) BARROS, V., V. CORDON, C. MOYANO, R. MENDEZ, J. FORQUERA, O. PIZZIO. Cartas de precipitación de la zona Oeste de las provincias de Río Negro y Neuquén. Universidad Nacional del Comahue, Centro Nacional Patagónico - CONICET. Argentina. 1983. 40 p.

(18) CORDON, V., J. FORQUERA, J. GASTIAZORO. Estudio microclimático del área cordillerana del SO de la provincia de Río Negro. Cartas de precipitación. Universidad Nacional del Comahue. Fac. Cs. Agrarias. Cinco Saltos, Río Negro. Argentina. 1993. 17p.

(19) DAVEL, M., A. ORTEGA. Estimación del índice de sitio para pino oregón a partir de variables ambientales en la Patagonia Andina Argentina. Bosque (Chile), 2003, vol. $24, \mathrm{~N}^{\mathrm{o}} 1$, p. 55-69.

(20) STAGE, A.R. An expression for the effect of aspect, slope and habitat type on tree growth. Forest Science, 1976, vol. 22, No 4, p. 457-460.

(21) CARVELL, K.L., E.H. TRYON. The effect of environmental factors on the abundance of oak regeneration beneath mature oak stands. Forest Science, 1961, vol. 7, No 2 .

(22) DRAPER, N.R., H. SMITH. Applied regression analysis. John Wiley \& Sons Inc. New York, USA. 1966. 709 p.

(23) KOLLMANN, F.F.P., W.A. COTÉ. Principles of wood science and technology. I Solid wood. Springer Verlag. Berlin. Germany, 1984, 592 p. 